\title{
Dendritic cells and vitamin D in dry eye disease
}

\author{
Balakrishnan .M1 , K. Sumathi2,", Pinky Karam³ ${ }^{3}$, Rajarathinam ${ }^{4}$ \\ ${ }^{1,2}$ Associate Professor, ${ }^{3}$ Post Graduate, ${ }^{4}$ Professor and HOD, ${ }^{2,3}$ Dept. of Biochemistry, ${ }^{1,4}$ Dept. of Ophthalmology, Sree Balaji \\ Medical College and Hospital, Bharath University, Chennai, Tamil Nadu, India
}

*Corresponding Author:

Email: sumathideepti@gmail.com

Received: $21^{\text {st }}$ January, 2018

Accepted: $28^{\text {th }}$ March, 2018

\begin{abstract}
To study the role of dendritic cells and vitamin D in troublesome evaporative dry eye diseases.

Materials and Methods: This is a cross sectional study which includes 108 patients having evaporative dry eye disease and 86 normal healthy volunteers. Ocular surface index, Shirmer's test and tear film break up time, corneal dendritic cell density, vitamin D and cytokines in serum/tear were estimated. Vitamin D, cytokine in serum/tear and corneal dendritic cell density were estimated.

Result: Higher dendritic cell density noticed in evaporative dry eye disease patients compared to control group volunteers and revealed positive correlation with ocular surface index $(\mathrm{P}<0.05)$. Inverse relationship was observed between ocular surface disease index and vitamin D and dendritic cell density. Inflammatory cytokine levels were increased significantly in evaporative dry eye patients.

Conclusion: Vitamin D has significant role in dry eye disease and its inflammation. Vitamin D therapy can be considered as an added treatment for dry eye disease.
\end{abstract}

Keywords: Dendritic cells, Vitamin D, Inflammatory cells, Dry eye.

\section{Introduction}

Dry eye refers to conditions were there is tear film instability either due to decreased tear production or increased tear evaporation resulting in discomfort in eye. ${ }^{1}$ The main and accessory lacrimal glands, meibomian glands, goblet cells of the conjunctiva, corneal and conjunctival surface epithelia and conjunctival vasculature all secrete a complex fluid mixture which constitutes the tear film which is a matrix like structure comprising of water, electrolytes, antimicrobial molecules, immunoglobulins, immunomodulators, growth factors like epithelial growth factor (EGF), fibrinogen, basic fibroblast growth factor (bFGF) neuropeptides and mucins. The cause for Dry Eye Disease (DED) is multifactorial. The ocular surface, the tear secreting glands, the neurological innervations and the various neuroendocrine factors all function as a single integrated unit. Any disorder in this unit will result in tear film instability resulting in ocular surface disease. In recent times it has been observed that the pathophysiology of dry eye leading to altered lacrimal gland tear production is related to neurohormonal and immune factors. $^{2} 25-40 \%$ of patients with meibomian gland dysfunction have dry eye of evaporative form. ${ }^{3}$ Dry eye otherwise called keratoconjunctivitis sicca involves two main categories. (a) hypo secretive which may be Sjogren or non Sjogren and (b) evaporative, (or) (c) both. Sjogren syndrome is a cytokine and receptor mediated inflammatory process that affects the lacrimal gland acini and ducts, leading to tear film instability resulting in OSD. Primary Sjogren syndrome is associated with xerostomia and the presense of antibodies indicative of autoimmune pathogenesis. Secondary Sjogren syndrome is characterized by systemic autoimmune connective tissue disorders like systemic lupus erythematosus, systemic sclerosis, rheumatoid arthritis, mixed connective tissue disease, dermatomyositis, primary biliary cirrhosis and relapsing polychondritis in addition to the features of primary Sjogren syndrome. Primary age related DED is most common in non Sjogren keratoconjunctivitis sicca. Other causes of DED are (a) lacrimal tissue destruction due to tumors or inflammation (e.g. thyroid eye disease, pseudo tumor and sarcoidosis) (b) absence of the lacrimal gland due to surgical removal or congenital. (c) lacrimal gland duct obstruction due to conjunctival scarring as seen in cicatricial pemphigoid or trachoma. (d) neurological lesions such as familial dysautonomia (Riley-Day Syndrome). Causes of evaporative conjunctivitis sicca are (a) oil deficiency secondary to obstructive meibomian gland dysfunction. (b) Tear film instability due to abnormal lid globe congruity or defective blinking. Some of the most common symptoms of dry eyes are foreign body sensation, irritation, stringy mucus discharge, transient blurring of vision, burning sensation, photophobia, itching and a tired or heavy feeling. In severe DED associated with filamentary keratitis, patients experience pain and irritation on blinking. However patients very rarely complain of a feeling of dryness in the eye and may present with complaints like lack of emotional tears or a decreased response to peeling or cutting of onions. The symptoms of DED are enhanced on conditions associated with increased tear evaporation like airconditioned environment, wind, central heating etc. (or) 
prolonged reading, watching television, computers with decreased blinking. However symptoms may improve following frequent lid closure.

Dry eye disease (DED) is very common worldwide with a prevalence rate of 5.5\%-33.7\%. ${ }^{4}$ Lot of research is going on worldwide regarding the etiopathogenesis, investigations and treatment modalities of DED to improve symptoms like pain, irritation, foreign body sensation, photophobia etc. where standard treatment methods failed in many cases presenting with severe DED. ${ }^{5}$ Many studies show an increased Dendritic Cell Density (DCD) in DED. ${ }^{6}$ Many etiologies have been implicated in the pathophysiology of dry eye. A fat soluble prohormone vitamin $\mathrm{D}$ has also been associated with DED. ${ }^{7,8}$ In our present study, the association between dendritic cell density and vitamin D level in relation to the severity in pain and irritation were analyzed.

\section{Materials and Methods}

This is a cross sectional study which was conducted on 104 patients having Evaporative Dry Eye disease (EDE) who came to eye department with presenting complaints of irritation, watering, pain and dimness of vision. There were 83 normal subjects selected as control volunteers. The study was conducted for 2 years from June 2015 to June 2017 analyzed in the department of ophthalmology along with department of biochemistry of sree balaji medical college in Chennai, India with tertiary eye care centre. Few patients were referred by general practitioners and outside general ophthalmologists, after conducting medical camps. Age, sex, history, vision and treatment outcome were noted. We followed Tenets of Declaration of Helsinki in this study. This study was approved by Institutional Ethics committee. In this study patients with eye diseases associated with dry eye disease were excluded.

Cornea was examined under slit lamp for dry eye signs. An increase in mucus strands and debris was noted in all patients. The lipid-contaminated mucin accumulated in the tear film and moved with each blink. The marginal tear meniscus was concave, small and in severe cases, it was absent. Punctuate epitheliopathy was documented in many cases. Filamentary keratits and mucus plaques were recorded in all cases. In all the patients tear film break-up time (BUT) was done to assess pre corneal tear film stability. In the lower fornix we instilled fluorescein. The patient was requested to blink several times and then stop. A broad beam and a cobalt blue filter were used to exam tear film. Appearance of black spots or lines denotes dry area. The BUT is the interval between the last blink and the formation of the first randomly distributed dry spot. An abnormal finding is appearance of BUT of less than 10 seconds. Rose Bengal test was done in all cases. Rose Bengal is a dye with an affinity for dead and devitalized epithelial cells and mucus. The typical staining pattern in dry eye consists of two triangles with their bases at the limbus. Corneal filaments and plaques were also shown up more clearly by the dye. Schirmer test also was done in all cases. The amount of wetting of $5 \mathrm{~mm}$ wide and $35 \mathrm{~mm}$ long in a Whatman filter paper by which this test was measured. This test was performed as follows. Gently dry the eye. The filter paper folded $5 \mathrm{~mm}$ from one end was inserted in the lower lid at the junction of middle and outer third. The patient was instructed with the eyes open and blink as necessary. The filter paper was removed fro the lower lid after 5 minutes and measured the amount of wetting. Over $15 \mathrm{~mm}$ measured in the filter paper was normal without topical anesthesia and slightly less with anesthesia. Less than $5 \mathrm{~mm}$ denotes impaired secretion and borderline between 5 and $10 \mathrm{~mm}$. Reading taken after topical anesthesia was due to basic secretion. Reading measured without topical anesthesia was due to basic and reflex secretion.

Shirmer's test values, Ocular Surface Disease Index (OSDI), and Tearfilm Break Up Time (TBUT) were taken as parameters and were evaluated for all subjects. Corneal DCD were taken in all subjects by In -Vivo Confocal Microscopy (IVCM) using Rostock Corneal Module/ Heidelberg Retina Tomography (RCM / HRTII; Heidelberg Engineering GmBH, Dossenheim, Germany). Tear/serum vitamin $\mathrm{D}$ and cytokines (Interlukin-17, IFNa, MCPI) were measured by flowcytometry based Cytometric-Bead-Array (CBA) and Enzyme Linked Immuno Sorbent Assay (ELISA) respectively. Patients were given anti dry eye treatment like tear substitute drops, gel, bandage soft contact lens, punctual plug and surgical procedures. We reviewed patients after 1 week, 2 weeks, 4 weeks, 6months and every year. Visual acuity recording, Slit lamp examination after fluorescein staining, and refraction were done for each patient during reviewal visit. Results were documented and analyzed at each visit.

Statistics: Dendritic cell density, ocular surface disease index, Shirmer's test, tear film break up time, tear/serum cytokines, tear/serum vitamin $\mathrm{D}$, were included as variables. We used descriptive statistics and percentages, mean and standard deviation were the data presented. Chi-square and Fishers exact test were used to test the difference in the two proportions. $\mathrm{P}<0.05$ was noted as statistically significant.

\section{Results}

Results of cross sectional 2 years long study of 104 patients and 86 normal subjects as control were analyzed. The following results were observed. The mean age of patients was $56 \pm 10$ year, 35:52 was the male: female ratio. $12 \pm 6$ months was mean follow up patients with diseases associated with it like exophthalmos, lid lag, facial palsy and ocular tumors were excluded from this study. Seventy eight (75\%) patients were selected from out patient department. Remaining twenty six (25\%) patients were referred by 
outside general practitioners and ophthalmologists after conducting medical camps.

Increased dendritic cell density observed in evaporative dry eye (EDE) disease patients on comparison to healthy volunteer controls showed positive correlation with ocular surface disease index $(\mathrm{P}<0.05)$. But decreased vitamin $\mathrm{D}$ observed in EDE patients compared to healthy controls presented with positive correlation with OSDI $(\mathrm{P}<0.05)$. So an inverse correlation was noted between OSDI values and vitamin $\mathrm{D}$ and dendritic cell density. Statistically significant higher levels of eye tear inflammatory agents like cytokines such as IL-17 (Interlukin), IFNa, MCPI were noted in evaporative dry eye patients. (Table 1)

Table 1: Results observed in evaporative dry eye disease

\begin{tabular}{|l|c|c|}
\hline \multicolumn{1}{|c|}{ Parameters } & Changes & P. Value \\
\hline DCD & Increased & $\mathrm{P}-<0.05$ \\
\hline Vitamin D & Decreased & $\mathrm{P}-<0.05$ \\
\hline Inflammatory cytokines & & \\
\hline a) Interlukin-17 & Increased & $\mathrm{P}-<0.05$ \\
\hline b) IFNa & Increased & $\mathrm{P}-<0.05$ \\
\hline c) MCPI & Increased & $\mathrm{P}-<0.05$ \\
\hline
\end{tabular}

DCD-Dendritic Cell Density

\section{Discussion}

Major challenge nowadays is dry eye disease management in ophthalmology. Many patients are ending at corneal opacity and blindness due to inefficient methods of treatment. So many innovative methods are evolving at various research levels. Continuous eye irritation, eye pain and watering in a small group of dry eye patients following standard and proper treatment strategies and the lack of tear film values to forecast such group of patients confront a major challenge in the therapy of dry eyes. So it is suggested to identify new diagnostic methods which can totally predict subjects whose symptoms and signs will not resolve with conventional treatment or may need additional food and environmental interventions along with topical therapy to make a good prognosis. The role of dendritic cells in modulation of nociception and pain has been studied earlier. ${ }^{9,10}$ Studies have described a possible role for corneal dendritic cells in the etiopathogenesis of dry eye, kerato conjunctivitis sicca and corneal allograft rejection. ${ }^{11,12}$ Inflammatory pathologies show an increase in the number of dendritic cells in the cornea. ${ }^{13}$ In our present study there was significant increase in dendritic cell density seen in evaporative dry eye disease has a positive association with the OSDI morbidity related sub scale results. Significant advances have been made in treating the dry eye, permanent cure is impossible so as to follow long term follow up with management. Tear supplementation so as to heal the dry eye by current therapy by which a normal ocular surface can be restored is now on focusing in improved treatment regime. The major drawback is inflammatory and cellular changes which impede healing as in chronic cases. So now they are focusing toward modulation of these aberrant process so as to improve and promote normal tear secretion by therapeutic approaches. Optimizing each layer, is central to the successful treatment of dry eyes (EDE) as the tear film is highly integrated unit. In evaporative dry eyes, stabilizing the lipid tear layer is considered as the primary treatment of tear evaporation. Since lipid tear substitutes are under trial and not widely available, it has be focused to improve the quality and quantity of native meibomain gland secretions. Meibomian gland secretion has been improved by lid hygiene, in the form of warm compress and lid massage. Seborrheic or bacterial load can be decreased by scrubbing the lids with dilute detergents so as to break the pro-inflammatory cycle of meibomian gland dysfunction. Systemic tetracycline has also been prescribed to treat local inflammation thereby meibomian gland function improving to make patient comfort relieved from symptoms. Chronic treatment to be continued for several weeks lower lid ptosis and lagophthalmos, due to increased exposure of the ocular surface due to eyelid abnormalities should be corrected can stabilize a decompensated ocular surface. Sometimes a partial or complete tarsorrhaphy or a conjunctival flap may be done in severe cases to prevent complete decompensation of the cornea. Evaporative pressure can be decreased by the use of humidifiers, moist chambers, glasses or goggles Dry eye patients with poor corneal wetting can be treated by new high-Dk, high-water-content contact lenses and new polymer lenses, accompanied by proper tear supplementation and hygiene.

There are various studies which have shown the association of dry eye diseases with vitamin D deficiency. ${ }^{14}$ Previous research had indicated vitamin D deficiency to be associated with neuralgia and chronic pain. ${ }^{15}$ In our study also there is strong inverse relation between vitamin D levels and the OSDI levels in EDE group. The role and mechanism of vitamin $\mathrm{D}$ level to pain remains a mystery but several theories are forwarded. Serotonin which can perpetuate chronic pain response was found to be in high levels in dry eye patients. ${ }^{16}$ Vitamin $\mathrm{D}$ is known to affect serotonin synthesis indicating the role of vitamin $\mathrm{D}$ in nociception. ${ }^{17}$ Vitamin $\mathrm{D}$ and its agonists have been found to inhibit maturation and induce tolerance in dendritic cells resulting in the arrest of inflammatory processes. ${ }^{18}$ Increased level of DCD with dendritic process (mature phenotype) were associated with an decreased vitamin D levels in our group. This support the present understanding regarding the vitamin D immunomodulatory function on dendritic cells. IL-17 has great role in inflammatory disorders and also has role in nociception by mediating mechanical allodynia by altering the expression of neuronal TRPV 4 channels essential for transduction of pain stimulus ${ }^{19}$ was found 
to be in increased levels in the tear film of patients. An increased IFNa, MCPI and ICAMI were observed in the patients as well. This could exacerbate the ocular symptoms, as they are reported to mediate pain. ${ }^{20-22}$

\section{Conclusion}

Our cross sectional study has many limitation. Our study had large sample size (104) and may be the biggest size. Our findings should be further strengthened by multiple studies with latest parameters and technologies. Our study shows that vitamin D levels is related to EDE manifestations and associated with inflammatory agents. The role of vitamin D supplement for the treatment of dry eye should be increased and researched further. We should get more details about the mechanism of involvement of vitamin $\mathrm{D}$ deficiency related to etiopathogenesis and abnormal inflammatory cytokines in tear film and eye health and eye pain for the management of dry eye.

\section{References}

1. Haas EB: The pathogenesis of kerato conjunctivitis sicca. Ophthalmologica. 1964;147:1-18.

2. Albert \& Jakobiecs: Priniciples and practice of ophthalmology, 3rd edition, Page 773-777.

3. Shields WJ, Mathers WD, Roberts J et al: Criteria for the evaluation of lid margin in blepharitis. Invest ophthalmol Vis Sci. 1990;31(suppl):483.

4. The epidemiology of dry eye disease: report of the Epidemiology Subcommittee of the International Dry Eye Workshop (2007). Ocul Surf. 2007 Apr;5(2):93-107.

5. Galor A, Batawi H, Felix ER, Margolis TP, Sarantopoulos KD, Martin ER, et al. Incomplete response to artificial tears is associated with features of neuropathic ocular pain. Br J Ophthalmol. 2016 Jun;100(6):745-9.

6. Kheirkhah A, Rahimi Darabad R, Cruzat A, Hajrasouliha AR, Witkin D, Wong N, et al. Corneal Epithelial Immune Dendritic Cell Alterations in Subtypes of Dry Eye Disease: A Pilot In Vivo Confocal Microscopic Study. Invest Ophthalmol Vis Sci. 2015 Nov;56(12):7179-85.

7. Galor A, Gardener H, Pouyeh B, Feuer W, Florez H. Effect of a Mediterranean dietary pattern and vitamin D levels on Dry Eye syndrome. Cornea. 2014 May;33(5):437-41.

8. Kurtul BE, Ozer PA, Aydinli MS. The association pf vitamin D deficiency with tear break-up time and Schirmer testing in non- Sjogren dry eye. Eye Lond Engl. 2015 Aug;29(8):1081-4.

9. Jeon Y-T, Na H, Ryu H, Chung Y. Modulation of Dendritic Cell Activation and Subsequent Th1 Cell Polarization by Lidocaine. PloS One. 2015;10(10):e0139845.
10. Luo J, Feng J, Liu S, Walters ET, Hu H. Molecular and cellular mechanisms that initiate pain and itch. Cell Mol Life Sci (CMLS). 2015 Sep;72(17):3201-23. doi: 10.1007/s00018-015-1904-4

11. Hattori T, Chauhan SK, Lee H, Ueno H, Dana R, Kaplan $\mathrm{DH}$, et al. Characterization of Langerin-expressing dendritic cell subsets in the normal cornea. Invest Ophthalmol Vis Sci. 2011 Jun;52(7):4598-604.

12. Gao N, Yin J, Yoon GS, Mi Q-S, Yu F-SX. Dendritic cell-epithelium interplay is a determinant factor for corneal epithelial wound repair. Am J Pathol. 2011 Nov; 179(5):2243-53.

13. Hamrah P, Liu Y, Zhang Q, Dana MR. Alterations in corneal stromal dendritic cell phenotype and distribution in inflammation. Arch Ophthalmol. 2003 Aug;121(8):1132-40.

14. Singman EL, Poon D, Jun AS. Putative corneal neuralgia responding to vitamin D supplementation. Case Rep Ophthalmol. 2013;4(3):105-8.

15. Shipton EE, Shipton EA. Vitamin D Deficiency and pain: Clinical Evidence of Low Levels of Vitamin D and Supplementation in Chronic Pain States. Pain Ther. 2015 Jun;4(1):67-87.

16. Chhadva P, Lee T, Sarantopoulos CD, Hackam AS. McClellan AL, Felix ER, et al. Human Tear Serotonin Levels Correlate with Symptoms and Signs of Dry Eye. Ophthalmology. 2015 Aug;122(8):1675-80.

17. Patrick RP, Ames BN. Vitamin D and the omega- 3 fatty acids control serotonin synthesis and action, part 2: relevance for ADHD, bipolar disorder, schizophrenia, and impulsive behavior. FASEB J. 2015 jun;29(6):2207-22.

18. Barragan M, Good M, Kolls JK. Regulation of Dendritic Cell Function by Vitamin D. Nutrients. 2015 Sep;7(9):8127-51.

19. Kim CF, Moalem-TaylorG.Interleukin-17 contributes to neuroinflammation and neuropathic pain following peripheral nerve injury in mice. J Pain Off J AM Pain Soc. 2011 Mar;12(3):370-83.

20. Tsuda M, Masuda T, Kitano J, Shimoyama H, TozakiSaitoh H, Inoue K. IFN-gamma receptor signaling mediates spinal microglia activation driving neuropathic pain. Proc Natl Acad Sci USA. 2009 May 12;106(19):8032-7.

21. Alvarez P, Green PG, Levine JD. Role for monocyte chemoattractant protein-1 in the induction of chronic muscle pain in the rat. Pain. 2014 Jun;155(6):1161-7.

22. Rodriguez Parkitna J, Korostynski M, KaminskaChowaniec D, Obara I, Mika J, Przewlocka B, et al. Comparison of gene expression profiles in neuropathic and inflammatory pain. J Physiol Pharmacol Off J Pol Physiol Soc. 2006 Sep;57(3):401-14.

How to cite this article: Balakrishnan $M$, Sumathi K, Karam P, Rajarathinam. Dendritic cells and vitamin $\mathrm{d}$ in dry eye disease. Int J Clin Biochem Res. 2018;5(3):431-434. 